Z Gerontol Geriat 2022 · 55:575-582 https://doi.org/10.1007/s00391-021-01972-x Eingegangen: 27. Januar 2021 Angenommen: 30. August 2021 Online publiziert: 29. September 2021 () Der/die Autor(en) 2021

\title{
Prädiktoren für die Nutzungsintensität von Tagespflegen bei Menschen mit kognitiven Einschränkungen
}

Catharina Wasić · Elmar Gräßel · Katharina Luttenberger · Carolin Donath

Zentrum für Medizinische Versorgungsforschung, Klinik für Psychiatrie und Psychotherapie, Universitätsklinikum Erlangen, Friedrich-Alexander-Universität Erlangen-Nürnberg (FAU), Erlangen, Deutschland

\section{Zusammenfassung}

Hintergrund: Tagespflegen sind etablierte Versorgungsangebote für Personen mit kognitiven Beeinträchtigungen. Die Nutzungsintensität ist mit durchschnittlich $3 \mathrm{~h} /$ Woche jedoch gering. Bisherige Studien konnten Prädiktoren für eine Nutzung/Nichtnutzung aufzeigen. Es ist jedoch bisher unklar, inwieweit diese Faktoren auch mit der Nutzungsintensität zusammenhängen.

Ziel der Arbeit: Identifikation von Prädiktoren für die Intensität der Tagespflegenutzung bei Menschen mit kognitiven Beeinträchtigungen, basierend auf dem Modell der Versorgungsinanspruchnahme nach Andersen.

Material und Methoden: Grundlage sind Daten der Studie Demenz in der Tagespflege bei psychosozialer MAKS-Intervention (DeTaMAKS). Prädiktoren für die Nutzungsintensität der Tagespflege wurden mit logistischer Regression analysiert. Ergebnisse: Eine signifikant höhere Intensität der Tagespflegenutzung lag vor bei: alleinlebenden Tagespflegegästen, höher gebildeten pflegenden Angehörigen, ab Pflegestufe 2, bei längerer bisheriger Nutzungsdauer der Tagespflege, bei vermehrten psychischen und Verhaltenssymptomen des Tagespflegegasts. Die Sensitivitätsanalyse zeigte bei zusammenlebenden Dyaden zusätzlich eine höhere Nutzungsintensität bei jüngeren pflegenden Angehörigen und kürzerer Entfernung zwischen Wohnort und Tagespflege, jedoch nicht in Bezug auf den Bildungsstand des pflegenden Angehörigen und die psychischen und Verhaltenssymptome des Tagespflegegasts.

Diskussion: Die Ergebnisse zeigen bestehenden Bedarf an Tagespflegeeinrichtungen, der sich durch Berufstätigkeit und das Leben ohne Partner erhöht. Neben einer guten Erreichbarkeit der Tagespflege könnten auch flexible Angebote die Intensität der Nutzung erhöhen.

\section{Schlüsselwörter}

Modell der Versorgungsinanspruchnahme $\cdot$ Demenz $\cdot \mathrm{MCl} \cdot$ Pflegende Angehörige $\cdot$ Regression

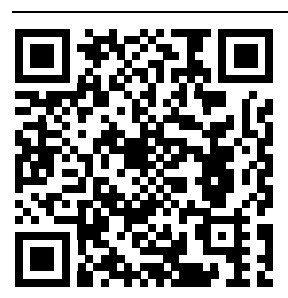

Tagespflegen entlasten pflegende Angehörige stundenweise durch die $\mathrm{Be}$ treuung eines zu Hause lebenden Pflegebedürftigen. Nur $4 \%$ der zu Hause lebenden Pflegebedürftigen in der Bundesrepublik Deutschland nutzen eine Tagespflegeeinrichtung [15] und dies durchschnittlich für $3 \mathrm{~h}$ die Woche [21]. Wovon hängt es $a b$, wie intensiv das Angebot der Tagespflege genutzt wird? Dieser Beitrag ermittelt Faktoren, die mit der Nutzungsintensität von Tages- pflegen im Zusammenhang stehen und ordnet sie in das Verhaltensmodell der Versorgungsinanspruchnahme nach Andersen et al. [1] ein.

\section{Hintergrund und Fragestellung}

\section{Tagespflegen in Deutschland}

Das Konzept der Tagespflege (TP) existiert in Deutschland seit 1973 [11] und ist durch $\S 41$ SGB XI als möglicher Bestandteil von 
Pflegeleistungen im Gesetz verankert [5]. Die Zahlen des Statistischen Bundesamtes zeigen, dass im Jahr 2019 82.639 Tagespflegeplätze in Deutschland zur Verfügung standen und 139.192 Pflegebedürftige dieses Angebot nutzten. Bei insgesamt 3,3 Mio. zu Hause lebenden Pflegebedürftigen besuchten somit ca. $4 \%$ davon eine TP [15].

Die TP zählt zu den bekanntesten formellen Hilfeleistungen. Laut einer Umfrage kannten 56,5\% der pflegenden Angehörigen die TP [10]. Bei einer Befragung von pflegenden Angehörigen einer Person mit Demenz war die TP die zweithäufigste genutzte Hilfeleistung [20].

Ein Review konnte zeigen, dass die Tagespflegenutzung für Personen mit Demenz eine Verbesserung des Schlafes und der Verhaltenssymptome und für ihre Angehörigen eine Verminderung der Pflegebelastung bedeuten kann [19]. Gleichzeitig war die Wahrscheinlichkeit eines Heimübertritts bei Nutzung einer TP signifikant erhöht, wenn keine anderen formellen Hilfeleistungen in Anspruch genommen wurden. Diese Ergebnisse konnten durch weitere, aktuelle Studien bestätigt werden [14, 16, 18].

\section{Nutzungsintensität von Tagespflegen: Rahmenkonzept}

Nach dem Verhaltensmodell der Versorgungsinanspruchnahme von Andersen et al. [1] bestimmen prädisponierende, ermöglichende und Bedarfsfaktoren die Nutzung eines Angebotes im Gesundheitssystem. Die Faktoren können dabei sowohl auf kontextueller Ebene, d.h. auf Ebene der Region, als auch auf individueller Ebene betrachtet werden. Prädisponierende Faktoren sind bestehende Merkmale einer Person bzw. Region, die eine mögliche Nutzung/Nichtnutzung beeinflussen. Die ermöglichenden Faktoren stellen Merkmale dar, die den Zugang zu Versorgungsangeboten begünstigen oder

\section{Abkürzungen}

\begin{tabular}{ll}
$M C l$ & $\begin{array}{l}\text { "Mild cognitive impairment“ (leichte } \\
\text { kognitive Beeinträchtigung) }\end{array}$ \\
$P A$ & Pflegende Angehörige \\
PmD & Person mit Demenz \\
$T P$ & Tagespflege \\
TP-Gäste & Tagespflegegäste \\
\hline
\end{tabular}

beeinträchtigen. Bedarfsfaktoren charakterisieren den Bedarf an Versorgung, der zu einer tatsächlichen Nutzung führt. In - Abb. 1 sind die bisherigen Forschungsergebnisse zur Nutzung von TP abgebildet und in Bezug auf das Andersen-Modell [1] eingeordnet.

Bisher ist jedoch unklar, inwieweit diese Faktoren auch mit der Nutzungsintensität von TP bei Menschen mit kognitiven Beeinträchtigungen zusammenhängen. Daraus ergab sich die Forschungsfrage: „Welche Faktoren des Verhaltensmodells der Versorgungsinanspruchnahme nach Andersen sind signifikante Prädiktoren für die Intensität der Tagespflegenutzung bei zu Hause lebenden Personen mit leichter kognitiver Beeinträchtigung $(\mathrm{MCl})$, leichter oder moderater Demenz?"

\section{Methodik}

\section{Design}

Die verwendeten Daten stammen aus der Studie Demenz in der Tagespflege bei psychosozialer MAKS-Intervention (DeTaMAKS-Studie), einer deutschlandweiten clusterrandomisierten kontrollierten Studie in Tagespflegeeinrichtungen. Ziel der Studie war der Wirksamkeitsnachweis einer nichtmedikamentösen Gruppentherapie für kognitiv beeinträchtigte Personen $(\mathrm{MCl}$, leichte oder moderate Demenz). Die Stichprobe bestand aus 453 Dyaden von Tagespflegegästen (TP-Gäste) und ihren pflegenden Angehörigen (PA). Alle TP-Gäste besuchten wenigstens für einen Tag/Woche eine TP. Informationen zu Studiendesign und -ablauf können dem Studienprotokoll entnommen werden [2]. Die Ergebnisse der primären Forschungshypothesen wurden bereits veröffentlicht $[3,8]$. Die Ethikkommission der Friedrich-Alexander-Universität Erlangen-Nürnberg hat der Durchführung der Studie inkl. datenschutzrechtlicher Aspekte zugestimmt (Votums-Nr.: 170_14B). Die Einverständniserklärungen wurden von allen teilnehmenden TP-Gästen und PA bzw. deren gesetzlicher Vertretung eingeholt. Die erhobenen Daten wurden ausschließlich pseudonymisiert gespeichert und ausgewertet. Die Studie wurde bei ISCRTN registriert (https://doi.org/10. 1186/ISRCTN16412551).
Die in diesem Beitrag verwendeten Querschnittsdaten stammen vom ersten Erhebungszeitpunkt vor dem Start der Therapie im April 2015.

\section{Stichprobe}

Von den 453 Dyaden, die an der DeTaMAKS-Studie teilnahmen, konnten die Daten von 449 Dyaden für die Hauptanalyse verwendet werden. (Bei 4 Dyaden fehlte die Angabe, wie häufig die TP genutzt wurde). Bestimmte ermöglichende Variablen (Entfernung und Fahrzeit zwischen Wohnung und TP, Regionstyp und Verstädterungsgrad der TP) lagen nur für zusammenlebende Dyaden vor. Daher wurde mit dieser Substichprobe $(n=275)$ eine Sensitivitätsanalyse durchgeführt.

Im Zusatzmaterial online finden sich die Charakteristika der Hauptstichprobe (Tabelle T1) sowie der Substichprobe (Tabelle T2). Die 34 an der Studie teilnehmenden TP wurden aus dem gesamten Bundesgebiet rekrutiert und sind in Tabelle T3 beschrieben.

\section{Instrumente}

Tabelle T4 gibt einen Überblick über die erfassten Variablen und genutzten Instrumente. Für eine genaue Beschreibung der verwendeten Instrumente: [2].

\section{Statistische Analysen}

Die Besuchshäufigkeit der TP in Tagen/ Woche stellte die abhängige Variable dar, dichotomisiert (angelehnt an Straubmeier et al. [17]) in Wenignutzung ( 1 bis 2 Tage/ Woche) und Häufignutzung ( 3 bis 5 Tage/ Woche).

Vor der statistischen Analyse wurden einzelne fehlende Werte in den unabhängigen Variablen mittels Regression imputiert. Die auszuwertende Hauptstichprobe umfasste 449 Fälle. Die Substichprobe umfasste 275 Fälle.

Die statistische Analyse erfolgte für die Hauptanalyse sowie für die Sensitivitätsanalyse in den gleichen drei Schritten:

I. Präanalyse: bivariater Vergleich nichtadjustiert

Es wurden Gruppenunterschiede zwischen Wenignutzern und Häufignutzern für alle potenziellen Prä- 


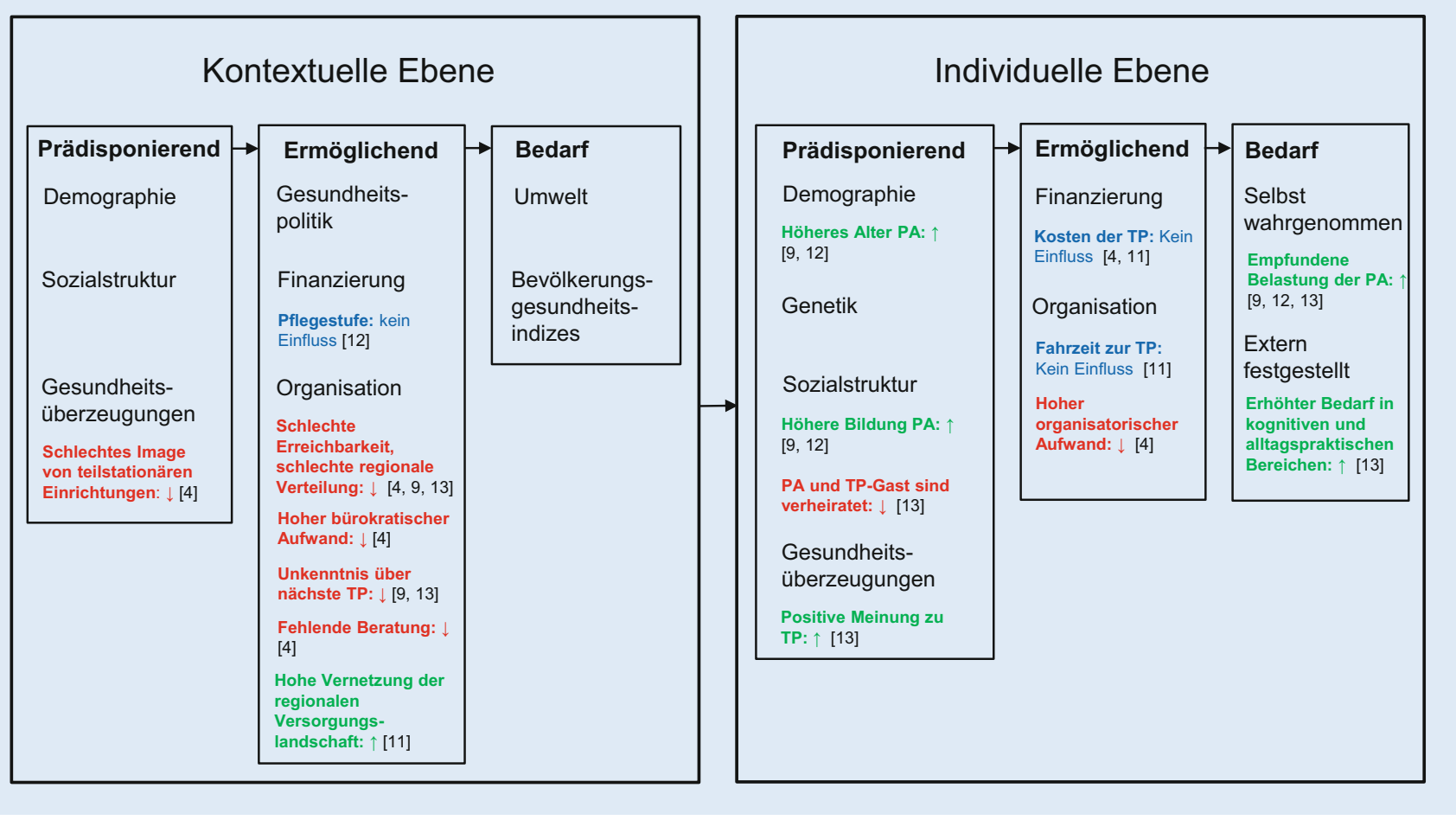

Abb. 1 \ Modell der Einflussfaktoren auf die Nutzungshäufigkeit von Tagespflege: Ergebnisse der empirisch-wissenschaftlichen Literatur. TP-Gast Tagespflegegast, PA pflegende Angehörige, TP Tagespflege, $\uparrow$ (grün) Nutzung nimmt zu, $\downarrow$ (rot) Nutzung nimmt ab, blau kein Einfluss. (Angelehnt an Andersen et al. [1] S. 35)

diktoren mit dem Chi-Quadrat-Test oder einem $\mathrm{t}$-Test für unverbundene Stichproben berechnet.

II. Multikollinearitätsprüfung:

Die unabhängigen Variablen, für die hinsichtlich der Nutzungsintensität entweder signifikante Gruppenunterschiede $(p<0,05)$ oder ein statistischer Trend $(p<0,1)$ bestanden, wurden auf Multikollinearität überprüft. Bei einem signifikanten Korrelationskoeffizienten über 0,5 wurden einzelne Variablen ausgeschlossen [6].

III. Finale Analyse: multivariates Modell adjustierte Analyse

Die nach Schritt II verbleibenden Variablen wurden als Prädiktoren in eine binär-logistische Regression mit Nutzungsintensität als abhängiger Variable (kodiert 0: Wenignutzung und 1: Häufignutzung) aufgenommen. Kategoriale Variablen mit mehr als 2 Ausprägungen wurden in eine dichotome Ausprägung umkodiert.

\section{Ergebnisse}

Hauptanalyse

I. Ergebnisse der Präanalyse Im Mittel wurde die TP an 2,29 Tagen besucht (SD: $\pm 1,29$ ). Damit sind $64,1 \%$ der Dyaden Wenignutzende und 35,9\% Häufignutzende von TP. Im Zusatzmaterial online, Tabelle T1, sind signifikante Unterschiede in 11 Variablen sowie 2 trendandeutende Variablen zwischen den beiden Nutzungsgruppen markiert, die in die Multikollinearitätsprüfung eingingen.

II. Ergebnisse der Multikollinearitätsprüfung

Es zeigte sich, dass das Alter der PA in einem starken Zusammenhang zur Berufstätigkeit der PA $(r=-0,648$, $p<0,001)$ und dem Verwandtschaftsgrad zum TP-Gast $(r=-0,641$, $p<0,001)$ stand. Aus Sparsamkeitsgründen wurde entschieden, die Variable Alter der PA beizubehalten.
III. Finale Analyse

Im finalen Modell resultierte aus der binär-logistischen Regressionsanalyse ein statistisch signifikantes Modell mit 5 signifikanten Prädiktoren $\left(\mathrm{X}^{2}=74,148\right.$ (df: 8); $\left.p<0,001\right)$. Nagelkerkes Pseudo- $R^{2}$ lag bei 0,21 , d. h., die untersuchten Variablen konnten $21 \%$ der Varianz der Nutzungsintensität erklären (• Tab. 1). Das finale Modell zeigte für folgende Faktoren eine signifikante Assoziation mit der Nutzungsintensität: Prädisponierende Faktoren waren der Familienstand der TP-Gäste (lebte der TP-Gast mit Partner/-in zusammen, sank die Wahrscheinlichkeit, die TP häufig zu nutzen) und der Bildungsstand der PA (mit jedem zusätzlichen Jahr mehr Bildung erhöhte sich die Wahrscheinlichkeit der Häufignutzung um ein Achtel). Ermöglichende Faktoren waren die Pflegestufe (Wahrscheinlichkeit der Häufignutzung war mehr als verdoppelt bei Pflegestufe 2 oder 3 im Vergleich zu einer niedrigeren oder keiner Pflegestufe) und die bisherige Dauer 
Tab. 1 Binär-logistische Regression der Hauptstichprobe mit Nutzungsintensität als abhängige Variable ( $n=449 ; 0$ : Wenignutzung, 1: Häufignutzung), Modell: Rückwärtsselektion

\begin{tabular}{|c|c|c|c|c|c|}
\hline & Unstd. B & $p$-Wert & Odds ratio & KI & Komponente \\
\hline Familienstand TP-Gast $^{\mathrm{a}}$ & $-0,889^{* *}$ & $<0,001$ & $0,411^{* *}$ & {$[-0,057-0,879]$} & Prädisponierend \\
\hline Bildungsstand (Jahre) PA & $0,122^{* *}$ & 0,001 & $1,129^{* *}$ & {$[1,057-1,202]$} & Prädisponierend \\
\hline Pflegestufe $^{b}$ & $0,987^{* *}$ & $<0,001$ & $2,683^{* *}$ & {$[2,210-3,156]$} & Ermöglichend \\
\hline Dauer der Tagespflegenutzung (Monate) & $0,018^{* *}$ & $<0,001$ & $1,018^{* *}$ & {$[1,008-1,029]$} & Ermöglichend \\
\hline NPI & $0,087^{*}$ & 0,038 & $1,091^{*}$ & {$[1,009-1,173]$} & Bedarf \\
\hline Inanspruchnahme einer Haushaltshilfe & $-0,480$ & 0,054 & 0,619 & {$[0,131-1,107]$} & Ermöglichend \\
\hline Inanspruchnahme eines häuslichen Betreuungsdienstes & $-0,650$ & 0,077 & 0,522 & {$[-0,199-1,244]$} & Ermöglichend \\
\hline NOSGER & 0,043 & 0,094 & 1,044 & {$[0,993-1,095]$} & Bedarf \\
\hline Konstante & $-3,285$ & $<0,001$ & 0,037 & {$[-1,217-1,212]$} & - \\
\hline \multicolumn{6}{|c|}{$\begin{array}{l}\text { Durch Selektion ausgeschlossene Variablen: Alter der pA, Inanspruchnahme eines ambulanten Pflegedienstes, Inanspruchnahme einer Angehörigenbera- } \\
\text { tungsstelle. Nagelkerkes Pseudo- } R^{2}=0,209 ; 4 \text { Schritte } \\
\text { Unstd. B unstandardisierter B-Koeffizient, KI Konfidenzintervall, TP-Gast Tagespflegegast, PA pflegende Angehörige } \\
{ }^{*} p<0,05,{ }^{* *} p<0,01 \\
\text { 'Dichotomisierte Variable Familienstand des TP-Gastes: mit Partner/-in zusammenlebend (verheiratet, in einer Beziehung): 1; ohne Partner/-in lebend (ledig, } \\
\text { getrenntlebend, geschieden, verwitwet): } 0 \\
{ }^{b} \text { Dichotomisierte Variable Pflegestufe: (keine Pflegestufe, Pflegestufe 0, Pflegestufe 1): 0, (Pflegestufe 2, Pflegestufe 3): } 1\end{array}$} \\
\hline
\end{tabular}

der Tagespflegenutzung (mit jedem zusätzlichen Monat der Nutzung erhöhte sich die Wahrscheinlichkeit der Häufignutzung um knapp 2\%). Bedarfsfaktoren waren psychische und Verhaltenssymptome (bei der Erhöhung des NPI um einen Punkt erhöhte sich die Wahrscheinlichkeit der Häufignutzung um 9\%).

\section{Sensitivitätsanalyse}

In zusammenlebenden Dyaden waren die TP-Gäste im Vergleich zur Hauptstichprobe jünger $(p=0,05)$ und häufiger männlich $(p=0,05)$, ihre PA jedoch im Mittel älter $(p=0,001)$, seltener berufstätig $(p=0,05)$, stärker durch die Pflegesituation belastet $(p=0,001)$ und nahmen seltener ambulante Pflegedienste und Essen auf Rädern in Anspruch (jeweils $p=0,05$ ).

I. Ergebnisse der Präanalyse

Die Substichprobe nutzte die TP im

Mittel an 2,22 Tagen (SD: $\pm 1,22$ ). $65,8 \%$ der Substichprobe gehörten zu den Wenignutzenden und 34,2\% zu den Häufignutzenden.

Im Zusatzmaterial online, Tabelle $\mathrm{T} 2$, sind signifikante Unterschiede in 9 Variablen sowie in 5 trendandeutenden Variablen zwischen den beiden Nutzungsgruppen markiert, die in die Multikollinearitätsprüfung eingingen.
II. Multikollinearitätsprüfung Bei der Prüfung auf Multikollinearität zeigte sich, dass 5 unabhängige Variablen stark untereinander korrelieren: Familienstand der TP-Gäste mit Geschlecht der TP-Gäste $(r=-0,543$, $p<0,001)$, Familienstand der TPGäste mit dem Verwandtschafts$\operatorname{grad}(r=0,600, p<0,001)$, Alter der PA mit dem Verwandtschaftsgrad $(r=-0,689, p<0,001)$, Alter der PA mit dem Familienstand der TP-Gäste ( $r=-0,522, p<0,001)$, Berufstätigkeit der PA mit dem Verwandtschaftsgrad $(r=0,549, p<0,001)$ und Berufstätigkeit der PA mit dem Alter der PA $(r=-0,708, p<0,001)$. Es wurden die Variablen Geschlecht der TP-Gäste und Alter der PA als ökonomischste Auswahl für die finale Analyse beibehalten.

III. Finale Analyse In der finalen Analyse resultierte aus der binär-logistischen Regressionsanalyse ein statistisch signifikantes Modell ( $x^{2}=62,846$ (df:7); $p<0,001$ ) mit 5 signifikanten Prädiktoren. Nagelkerkes Pseudo- $R^{2}$ lag bei 0,28. Die in der Sensitivitätsanalyse untersuchten Variablen erhöhen die aufgeklärte Varianz der Nutzungsintensität somit auf $28 \%$ (•Tab. 2).

Das finale Modell zeigte analog zur gesamten Stichprobe den Zusammenhang der Pflegestufe und der bisherigen Dauer der Tagespflege- nutzung mit der Nutzungsintensität. Zusätzlich kamen als prädisponierende Faktoren das Alter der PA (mit jedem Jahr, das die PA älter waren, verringerte sich die Wahrscheinlichkeit der Häufignutzung), der Familienstand der PA (bei PA, die mit Partner/-in zusammenlebten, sank die Wahrscheinlichkeit der Nutzung) sowie als ermöglichender Faktor die Entfernung zwischen Wohnort und TP (mit jedem Kilometer mehr verringerte sich die Wahrscheinlichkeit der Häufignutzung) hinzu.

Eine Zuordnung der in dieser Studie erhobenen unabhängigen Variablen in das Verhaltensmodell der Versorgungsinanspruchnahme nach Andersen et al. [1] sowie deren Einfluss auf die Intensität der Tagespflegenutzung zeigt • Abb. 2.

\section{Diskussion}

Ziel der Analyse war es, Prädiktoren für die Nutzungsintensität von TP bei Personen mit kognitiver Beeinträchtigung und ihren PA zu finden. Dabei stellten sich der Familienstand des TP-Gastes, der Bildungsstand der PA, die Pflegestufe, die bisherige Dauer der Tagespflegenutzung, psychische und Verhaltenssymptome (NPI) sowie bei zusammenwohnenden Dyaden zusätzlich das Alter und der Familienstand der PA und die Entfernung zur TP als signifikante Prädiktoren heraus. 
Tab. 2 Binär-logistische Regression der Substichprobe mit Nutzungsintensität als abhängige Variable bei zusammenwohnenden Dyaden $(n=275 ; 0$ : Wenignutzung, 1: Häufignutzung)

\begin{tabular}{|c|c|c|c|c|c|}
\hline & Unstd. B & $p$-Wert & Odds ratio & KI & Komponente \\
\hline Alter des PA & $-0,030^{*}$ & 0,020 & $0,971^{*}$ & {$[0,946-0,996]$} & Prädisponierend \\
\hline Familienstand des $\mathrm{PA}^{\mathrm{a}}$ & $-0,826^{*}$ & 0,022 & $0,438^{*}$ & {$[-0,267-1,142]$} & Prädisponierend \\
\hline Pflegestufe $^{b}$ & $0,945^{* *}$ & 0,001 & $2,573^{* *}$ & {$[2,001-3,146]$} & Ermöglichend \\
\hline Dauer der Tagespflegenutzung (Monate) & $0,029^{* *}$ & $<0,001$ & $1,030^{* *}$ & {$[1,014-1,045]$} & Ermöglichend \\
\hline Entfernung $(\mathrm{km})^{\mathrm{c}}$ & $-0,078^{*}$ & 0,010 & $0,925^{*}$ & {$[0,866-0,984]$} & Ermöglichend \\
\hline Angehörigenberatungsstelle & $-0,923$ & 0,080 & 0,397 & {$[-0,638-1,432]$} & Ermöglichend \\
\hline Haushaltshilfe & $-0,666$ & 0,053 & 0,514 & {$[-0,160-1,188]$} & Ermöglichend \\
\hline Konstante & 1,459 & 0,077 & 4,301 & {$[2,684-5,918]$} & - \\
\hline \multicolumn{6}{|c|}{$\begin{array}{l}\text { Durch Selektion ausgeschlossene Variablen: Geschlecht des Tagespflegegastes, Dauer der häuslichen Pflege, Inanspruchnahme eines häuslichen Betreuun } \\
\text { dienstes, Inanspruchnahme einer Betreuungsgruppe. Nagelkerkes } R^{2}=0,282 ; 5 \text { Schritte } \\
\text { Unstd. B unstandardisierter B-Koeffizient, KI Konfidenzintervall 95, PA pflegende Angehörige } \\
{ }^{*} \boldsymbol{p}<0,05,{ }^{* *} p<0,01 \\
\text { 'Dichotomisierte Variable Familienstand des PA: mit Partner/-in zusammenlebend (verheiratet, in einer Beziehung): 1; ohne Partner/-in lebend (ledig, ge- } \\
\text { trenntlebend, geschieden, verwitwet): } 0 \\
\text { 'Dichotomisierte Variable Pflegestufe: (keine Pflegestufe, Pflegestufe 0, Pflegestufe 1): 0, (Pflegestufe 2, Pflegestufe 3): } 1 \\
\text { 'Zwischen Tagespflege und Wohnort }\end{array}$} \\
\hline
\end{tabular}

\section{Prädisponierende Faktoren}

Bei Donath et al. und Lüdecke et al. [8, 12] war ein höheres Alter der PA mit der Nutzung von TP assoziiert. Die vorliegende Analyse zeigte bei zusammenlebenden Dyaden jedoch den gegenteiligen Zusammenhang. Dies lässt sich möglicherweise dadurch erklären, dass die jüngeren PA der hier untersuchten Dyaden fast ausschließlich Kinder/Schwiegerkinder, eher berufstätig und somit eher auf eine Betreuung angewiesen waren als die älteren PA, die zumeist Partner/-in des jeweiligen TP-Gastes waren. Dies passt auch zu dem Befund, dass eine TP weniger intensiv genutzt wurde, wenn der TP-Gast in einer Partnerschaft lebt.

Übereinstimmend mit der Literatur [9, $12,13]$ zeigen auch die vorliegenden Ergebnisse, dass eine höhere Bildung der PA mit einer höheren Nutzungsintensität korreliert.

\section{Ermöglichende Faktoren}

Der hier gefundene Zusammenhang von erhöhter Nutzung bei höherer Pflegestufe bestätigt sich in der Literatur nicht [4, 12]. Im System der Pflegestufen wurden die Kosten für die TP mit anderen Leistungen der Pflegekasse verrechnet. Diese Verrechnung wurde erst kurz vor Studienbeginn aufgegeben. Eine erhöhte Nutzungsintensität bei Vorliegen höherer Pflegestufen lässt sich somit nur eingeschränkt durch bessere Finanzierungsmöglichkeiten erklären - insbesondere für Dyaden, die die TP schon länger nutzten. Stattdessen wird dies als erhöhter Bedarf interpretiert. Allerdings kann nicht von einem linear steigenden Bedarf ausgegangen werden, da Personen mit höchster Pflegestufe der Besuch einer TP meist nicht mehr möglich ist. Dies zeigt sich auch darin, dass in der hier untersuchten Stichprobe nur 4 Personen die Pflegestufe 3 hatten.

Mit längerer bereits bestehender Nutzung stieg die Intensität der Nutzung der TP. Dies ist ein Hinweis darauf, dass die Nutzung von den PA und/oder den TPGästen nach einer Eingewöhnungsphase als positiv empfunden wird. Es scheint eine Anfangshürdezu geben, diezu Beginn eine niedrigere Nutzung mit sich bringt. Derzeit liegen dazu jedoch keine hinreichenden, wissenschaftlichen Erkenntnisse vor. Die beiden ermöglichenden Faktoren Pflegestufe und Nutzungsdauer zeigten sich sowohl in der Haupt- als auch in der Sensitivitätsanalyse als signifikante Prädiktoren, was für eine Belastbarkeit dieser Befunde spricht.

Die Entfernung zur TP als signifikanter ermöglichender Faktor in der Substichprobe deckt sich mit Donath et al. und Phillipson et al. $[8,13]$ bezüglich der Nutzung/ Nichtnutzung, jedoch nicht mit KremerPreiß [11]. Sind die PA selbst für die Organisation des Transportes zuständig, werden Fahrten mit größerer Entfernung zeitaufwendiger, schwieriger und im Fall von stark ausgeprägten psychischen und Verhaltenssymptomen bei den TP-Gästen anstrengender. Einige TP bieten einen Transport der TP-Gäste an. Diese Information wurde nicht erhoben, jedoch ist es plausibel, dass der Einfluss der Entfernung zur TP bei einem Transportangebot sinkt.

\section{Bedarfsfaktoren}

Die einzigen signifikanten Bedarfsfaktoren in der Hauptstichprobe waren psychische und Verhaltenssymptome. Bei zusammenwohnenden Dyaden zeigte sich dieser Zusammenhang nicht. Dies ist erstaunlich, da gerade die psychischen und Verhaltenssymptome das Zusammenleben stark belasten können [7]. Die von anderen Autoren $[8,12,13]$ identifizierten Zusammenhänge von Tagespflegenutzung und Belastung der PA bzw. den Einschränkungen der TP-Gäste in kognitiven und alltagspraktischen Bereichen konnten in Bezug auf die Nutzungsintensität nicht gefunden werden. Allerdings wurden nur Dyaden in die Studie eingeschlossen, die die TP bereits nutzten. Es ist möglich, dass sich in dieser Studie Bedarfsfaktoren nicht hinreichend identifizieren ließen, da ein Bedarf bei allen Nutzern vorhanden und damit die Varianz diesbezüglich in der Stichprobe gering war. Somit wäre ein Zusammenhang zwischen Nutzungsintensität und Bedarf nicht abbildbar. 


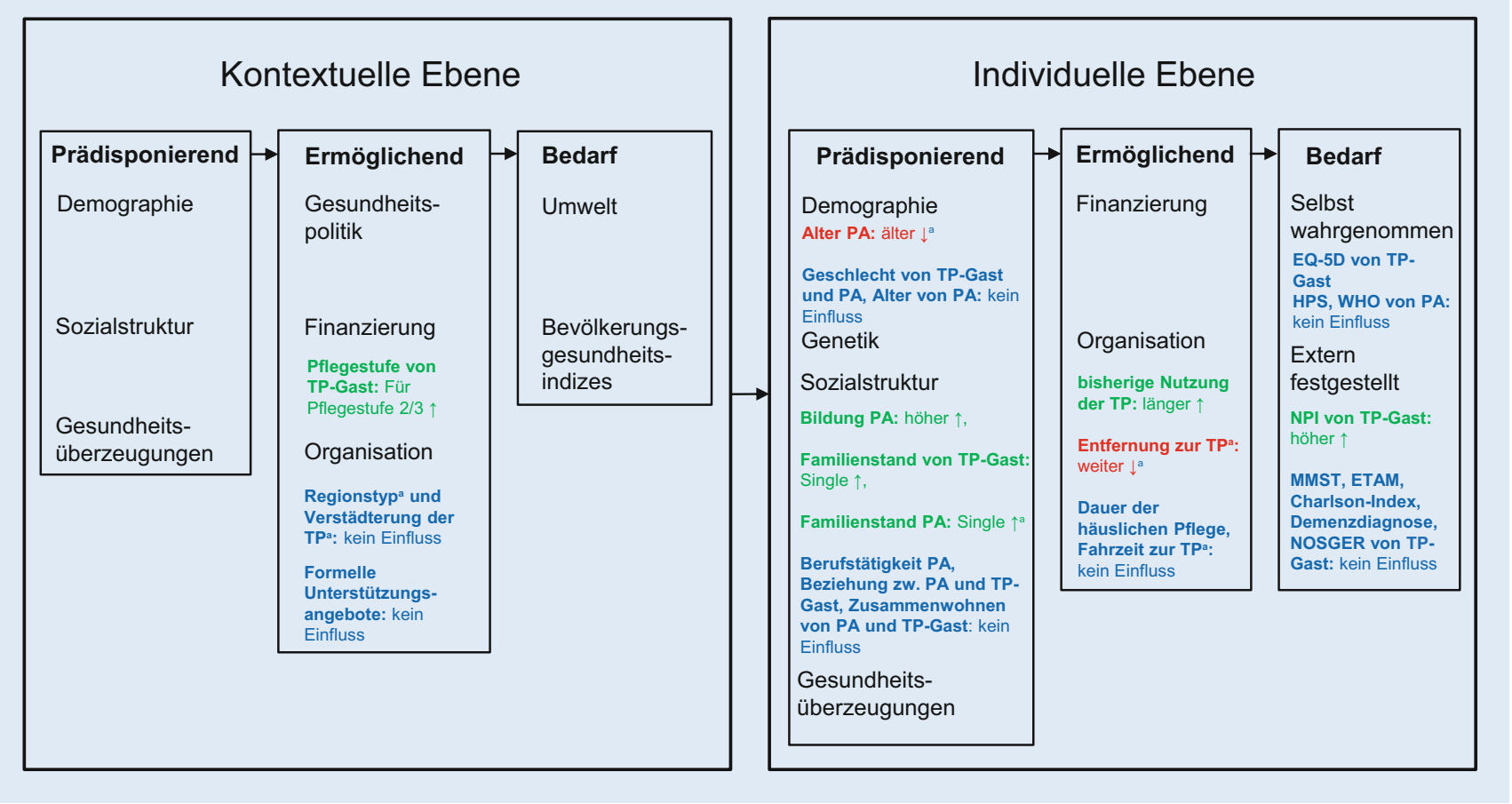

Abb. 2 ॥ Operationalisierung des Modells der Versorgungsinanspruchnahme von Andersen in dieser Studie mit Ergebnissen. TP-Gast Tagespflegegast, PA pflegende Angehörige, TP Tagespflege, ${ }^{a}$ Daten nur für eine Substichprobe vorhanden, $\uparrow$ (grün) Nutzung nimmt zu, $\downarrow$ (rot) Nutzung nimmt ab, blau kein Einfluss. (Angelehnt an Andersen et al. [1] S. 35)

\section{Limitationen}

Mit den verwendeten Daten können keine Rückschlüsse auf Nichtnutzende von TP gezogen werden, weil die Stichprobe nur aus Nutzenden von TP bestand. Es handelte sich außerdem um Querschnittsdaten, die keinen kausalen Schluss ermöglichen. Die Daten zu Wohnort sowie räumlicher und zeitlicher Entfernung zur TP konnten nur für einen Teil der Stichprobe erhoben werden. Da der Erhebungszeitpunkt der Daten vor der letzten Reform des Pflegeversicherungsgesetzes lag, waren die Leistungen für die Inanspruchnahme von TP in der vorliegenden Stichprobe deutlich eingeschränkter als gegenwärtig. Zieht man die aktuellen Leistungen für TP in Betracht, ist ein noch stärkerer Einfluss des Pflegegrades auf die Nutzungsintensität der TP anzunehmen, als hier festgestellt wurde. Einige Faktoren des Verhaltensmodells der Versorgungsinanspruchnahme wie Gesundheitspolitik, Umwelt oder Angebotsflexibilität sowie Gesundheitsüberzeugungen oder Genetik konnten auf Basis der verwendeten Daten nicht untersucht werden. Zukünftige Studien könnten diese sowie die Abhängigkeiten zwischen verschiedenen Faktoren in den Blick nehmen.

\section{Fazit für die Praxis}

- Der Bedarf an Betreuung und Förderung durch Tagespflegen ist vorhanden und steigt mit zunehmender Pflegebedürftigkeit.

- Der Bedarf wird in Zukunft durch die verbreitete Berufstätigkeit aller Bevölkerungsgruppen und Lebensformen auBerhalb von festen Partnerschaften eher zunehmen.

- Die Entfernung der Tagespflege ist ein signifikanter Prädiktor für die Nutzungsintensität. Um die Nutzung zu erhöhen, müssten Tagespflegen für potenzielle Nutzende schnell erreichbar sein.

- Pflegende Angehörige und Menschen mit kognitiven Beeinträchtigungen sollten die Möglichkeit bekommen, das Angebot zunächst probeweise kennenzulernen. Als Erleichterung des „Einstiegs“ könnten Einrichtungen eine "Testwoche"/einen "Testmonat" anbieten. Auch flexible Nutzungskonzepte mit z. B. Zeitkontingenten könnten eine nachfolgend stärkere Nutzungsintensität fördern. 
torgrades „Dr. rer. biol. hum." von Catharina Wasic durchgeführt.

Förderung. Die vorliegende Studie wurde finanziert durch Forschungsgelder des Spitzenverbands der Gesetzlichen Krankenkassen Deutschlands (GKVSpitzenverband, Deutschland) und des Bayerischen Staatsministeriums für Gesundheit und Pflege, Deutschland.

Funding. Open Access funding enabled and organized by Projekt DEAL.

\section{Einhaltung ethischer Richtlinien}

Interessenkonflikt. C. Wasić, E. Gräßel, K. Luttenberger und C. Donath geben an, dass kein Interessenkonflikt besteht.

Alle beschriebenen Untersuchungen am Menschen wurden mit Zustimmung der zuständigen EthikKommission, im Einklang mit nationalem Recht sowie gemäß der Deklaration von Helsinki von 1975 (in der aktuellen, überarbeiteten Fassung) durchgeführt. Von allen beteiligten Personen (TP-Gast und pA) liegt eine Einverständniserklärung vor.

Open Access. Dieser Artikel wird unter der Creative Commons Namensnennung 4.0 International Lizenz veröffentlicht, welche die Nutzung, Vervielfältigung, Bearbeitung, Verbreitung und Wiedergabe in jeglichem Medium und Format erlaubt, sofern Sie den/die ursprünglichen Autor(en) und die Quelle ordnungsgemäß nennen, einen Link zur Creative Commons Lizenz beifügen und angeben, ob Änderungen vorgenommen wurden.

Die in diesem Artikel enthaltenen Bilder und sonstiges Drittmaterial unterliegen ebenfalls der genannten Creative Commons Lizenz, sofern sich aus der Abbildungslegende nichts anderes ergibt. Sofern das betreffende Material nicht unter der genannten Creative Commons Lizenz steht und die betreffende Handlung nicht nach gesetzlichen Vorschriften erlaubt ist, ist für die oben aufgeführten Weiterverwendungen des Materials die Einwilligung des jeweiligen Rechteinhabers einzuholen.

Weitere Details zur Lizenz entnehmen Sie bitte der Lizenzinformation auf http://creativecommons.org/ licenses/by/4.0/deed.de.

\section{Literatur}

1. Andersen RM, Davidson PL, Baumeister SE (2014) Improving access to care. In: Kominski GF (Hrsg) Changing the U.S. health care system. Jossey-Bass, San Francisco, S 33-69

2. Behrndt E-M, Straubmeier M, Seidl H et al (2017) The German day-care study: multicomponent non-drug therapy for people with cognitive impairment in day-care centres supplemented with caregiver counselling (DeTaMAKS)—study protocol of a cluster-randomised controlled trial. BMC Health Serv Res 17:492

3. Behrndt EM, Straubmeier $M$, Seidl $H$ et al (2019) Brief telephone counselling is effective for caregivers who do not experience any major life events-caregiver-related outcomes of the German day-care study. BMC Health Serv Res 19:20

\section{Predictors of intensity of use of adult day care centers in people with cognitive impairment}

Background: Adult day care is an established concept in Germany for people with cognitive impairment; however, only a small fraction of people in need for care actually use adult day care. Studies so far highlighted some predictors for the use of adult day care; however, it remains unclear which factors are associated with the intensity of use. Objective: To identify relevant predictors for the intensity of use of adult day care using the Andersen healthcare utilization model.

Material and methods: Data used were obtained within the project dementia in day care with psychosocial MAKS interventions (DeTaMAKS), which studied adult day care users with cognitive impairments and their family caregivers. A logistic regression was performed to predict frequent or low use of adult day care.

Results: The following factors were significantly associated with higher intensity of use: civil status of adult care user being widowed or single, higher educational level of caregiver, higher care level, longer duration of adult day care use and more mental and behavioral symptoms of the adult day care user. The sensitivity analysis for cohabiting dyads additionally showed a higher intensity of use with a lower age of the caregiver and shorter distance between place of residence and adult day care but not with respect to educational level of the caregiver and mental and behavioral symptoms of the user.

Conclusion: The results show a need for adult day care, which increases with caregivers being employed and users living outside of permanent relationships. A short distance to the adult care center as well as flexible care options may increase the frequency of use.

\section{Keywords}

Healthcare utilization model · Dementia · Cognitive dysfunction · Family caregivers · Regression

4. Büker C, Niggemeier M (2014) Tagespflege für ältere Menschen - Entstehung und Entwicklung der Tagespflege. Kohlhammer, Stuttgart

5. Bundesministerium der Justiz (1994)DasElfteBuch Sozialgesetzbuch - Soziale Pflegeversicherung (Artikel 1 des Gesetzes vom 26. Mai 1994, BGBI. I S. 1014, 1015), das zuletzt durch Artikel 2 des Gesetzes vom 21. Dezember 2019 (BGBI. IS. 2913) geändert worden ist

6. Cohen J (1992) A power primer. Psychol Bull 112:155-159

7. Dietzel N, Karrer L, WolffFet al (2020) Einflussfaktoren auf die Pflegebelastung der Angehörigen von Menschen mit Demenz: der Bayerische Demenz Survey (BayDem). Gesundheitswesen 82:30-39

8. Donath C, Luttenberger K, Graessel E et al (2019) Can brief telephoneinterventions reduce caregiver burden and depression in caregivers of people with cognitive impairment?-long-term results of the german day-care study (RCT). BMC Geriatr 19:1-15

9. Donath C, Winkler A, Graessel E et al (2011) Day care for dementia patients from a family caregiver's point of view: a questionnaire study on expected quality and predictors of utilisation - part II. BMC Health Serv Res 11:76

10. Frey $C$, Heese $C$ (2011) Versorgung und Hilfe bei Demenz-BekanntheitvonEntlastungsangeboten für Angehörige und Versorgungswünsche. Pflege Ges 16:271-282

11. Kremer-Preiß U (1998) 20 Jahre Erfahrungen mit Tagespflegeeinrichtungen. TUP 7:248-253

12. Lüdecke D, Mnich E, Kofahl C (2014) How do socioeconomic factors influence the amount and intensity of service utilization by family caregivers of elderly dependents? In: Janssen C,
Swart E, Lengerke T(Hrsg) Health care utilization in Germany. Springer, New York, S171-189

13. Phillipson L, Jones SC, Magee C (2014) A review of the factors associated with the non-use of respite services by carers of people with dementia: implications for policy and practice. Health $\mathrm{Soc}$ Care Community 22:1-12

14. Rokstad AMM, Engedal K, Kirkevold $\varnothing$ et al (2018) The impact of attending day care designed for home-dwelling people with dementia on nursing home admission: a 24-month controlled study. BMC Health Serv Res 18:864

15. Statistisches Bundesamt (2020) Pflegestatistik 2019. Pflege im Rahmen der Pflegeversicherung. Deutschlandergebnisse. DeStatis, Wiesbaden

16. Strandenæs MG, Lund A, Rokstad AMM (2018) Experiences of attending day care services designed for people with dementia-a qualitative study with individual interviews. Aging Ment Health 22:764-772

17. Straubmeier M, Behrndt E-M, Seidl $H$ et al (2017) Non-pharmacological treatment in people with cognitive impairment-results from the randomized controlled German Day Care Study. Dtsch Arztebl Int 114:815-821

18. Tretteteig S, Vatne S, Rokstad AM (2017) The influence of day care centres designed for people with dementia on family caregivers-a qualitative study. BMC Geriatr 17:5

19. Vandepitte S, Van Den Noortgate N, Putman K et al (2016) Effectiveness of supporting informal caregivers of people with dementia: a systematic review of randomized and non-randomized controlled trials. J Alzheimers Dis 52:929-965

20. von Kutzleben M, Reuther S, Dortmann $O$ et al (2016) Care arrangements for community- 
dwelling people with dementia in Germany as perceived by informal carers-a cross-sectional pilot survey in a provincial-rural setting. Health Soc Care Community 24:283-296

21. Zank S, Schacke C, Leipold B (2007) Längsschnittstudie zur Belastung pflegender Angehöriger von demenziell Erkrankten (LEANDER). GeroPsych 20:239-255

\section{â. \\ A-IMA \\ Ambulantes \\ Interdisziplinär-Multimodales \\ Assessment}

\section{Ambulantes Interdisziplinäres Multimodales Assessment (A-IMA) startet in Deutschland}

Die Deutsche Schmerzgesellschaft e.V. hat ein neues präventives ambulantes Untersuchungsangebot entwickelt, um eine Chronifizierung alltäglicher Kopf-, Rücken- oder Gelenkschmerzen zu verhindern. Dieses Angebot ermöglicht es, frühzeitig und berufsgruppenübergreifend die Ursache für anhaltende Schmerzen herauszufinden und eine geeignete Therapie einzuleiten. Das sogenannte Ambulante Interdisziplinäre Multimodale Assessment (A-IMA) wird zunächst an 25 deutschen Zentren eingeführt.

„Zu handeln, bevor Schmerzen chronisch werden, ist nötig und möglich“, erklärt Professor Dr. Winfried Meißner, Präsident der Deutschen Schmerzgesellschaft e.V. Notwendig ist dafür idealerweise die Expertise unterschiedlicher Berufsgruppen: Schmerzgeplagte sollten möglichst ärztlich, psychologisch und physiotherapeutisch untersucht werden, um ein Gesamtbild des Risikos einer Chronifizierung zu erhalten. Die Deutsche Schmerzgesellschaft hat den Nutzen eines solchen Interdisziplinären Multimodalen Assessments (IMA) bereits im Rahmen des Projekts PAIN2020 wissenschaftlich untersucht. „Mit der ambulanten Form dieser Behandlung - A-IMA - überführen wir nun konsequenterweise zentrale Inhalte der Studie in die Patientenversorgung in Deutschland", sagt Meißner.

Im Rahmen von A-IMA erhalten die Betroffenen bereits nach sechs Wochen anhaltender Schmerzen in spezialisierten schmerztherapeutischen Zentren die Möglichkeit, in einem eintägigen Assessment umfassend untersucht zu werden. Das Team aus Ärzten, Psychologen und Physiotherapeuten stellt dann eine gemeinsame Diagnose und gibt eine fundierte, Leitlinien-konforme Therapieempfehlung. Der Therapieplan wird im Anschluss gemeinsam mit dem Patienten erstellt. Derzeit laufen Verhandlungen mit den Krankenkassen, um möglichst vielen Versicherten dieses Angebot zu ermöglichen.

In einem anderen Projekt („POET Pain“), das ebenfalls im Rahmen von PAIN2020 gefördert wird, geht es um die Prävention operationsbedingter anhaltender Schmerzen. Denn auch bei Schmerzen nach einer Operation besteht die Gefahr, dass diese chronifizieren. Das hängt vor allem vom individuellen Risikoprofil der Betroffenen sowie den Maßnahmen und Strukturen der Nach-OP-Versorgung ab. Die Schmerzgesellschaft wird einen sogenannten „Transitional Pain Service" aufbauen, der die besonders gefährdeten Patient*innen identifiziert und geeignete interdisziplinäre Nachbetreuung sicherstellt. 